\title{
Design of Quality Inspection System for Red Dates
}

\author{
Ren Zhang,Pengcheng Zhang,Hongbing Meng* \\ College of Information Engineering, Tarim University, Alar, Xinjiang, \\ 843300
}

\begin{abstract}
In this paper, the near infrared spectroscopy analysis technology, combined with the traditional appearance of quality testing and chemical analysis methods, the establishment of red date quality characteristics of different quantitative digital calibration model. Based on the accumulated data of red date quality, the quality of red date such as sugar content, water content, color, texture and so on were digitized and quantitatively described, and the software of multi-parameter digital detection and evaluation of red date quality in Nanjiang was completed. And through the promotion and application in the enterprise gradually improve the quality of southern Xinjiang red date detection model. And lay the foundation for digitization and informatization of quality detection of red date in southern Xinjiang.

Keywords:multi-objective bilevel programming, genetic algorithm, interpolation.
\end{abstract}

\section{Introduction}

Red dates, also known as red date. Is characterized by very high vitamin content, "natural vitamin pills" in the world, with yin and yang, the effect of blood. Red date is known as "hardcore crops," said, with drought, water logging-resistant characteristics, is the development of water-saving fruit and vegetable industry preferred varieties. Therefore, the quality of red date detection is particularly important, and now the identification of the quality of red date pure or botanical principles to use more detection, the cumbersome steps, detection is slow, to go through many trials and tests, can not be accurate and efficient In view of the 
above-mentioned problems, the present invention devises a red date quality detection system to solve the above-mentioned problems.

\section{Red date detection system structure and principle analysis}

The red date quality detection system comprises a central processing unit, an internal testing subsystem, an external detecting subsystem, an output/input device and a wireless transmission subsystem. The quality control system of the red date is detected by an internal detecting subsystem and an external detecting subsystem Sugar content, anthocyanin content and color, longitudinal diameter, transverse diameter, shape, weight and other characteristics of the red dates of the protein content, titratable acid content, reducing sugar content, and through the wireless transmission subsystem to the database for data Contrast, and finally through the display, can effectively filter the red date, simple structure, low production costs, which will help promote the use of, and through the magnetic field lines to the principles of red dates can be quickly detected within the red dates, and after testing the signal Output for subsequent analysis.

The internal detection subsystem comprising a detection sensor, an excitation element, an excitation driver, a power supply module, a receiver, an amplifier and an analog-to-digital converter, the excitation element being connected to the receiver input, the receiver output being connected to an amplifier, Connected to the power supply module and the excitation element, the input end of the digital converter is connected with the amplifier, and the output end of the analog-todigital converter is connected with the central processing unit. The detection sensor is in close contact with the outer surface of the red date, and the receiver receives the detection sensor And the receiver transmits the received signal to the amplifier to amplify and amplify the amplified signal to output the signal amplified by the amplifier to convert the digital-to-analog signal into an analogto-digital converter for transmission to the central processing unit to analyze and detect the red dates The contents of protein, titratable acid, reducing sugar, sucrose and anthocyanin.

The external detection subsystem comprises a camera, a gravity sensor, a data acquisition unit, a data processing unit, a number of electric signal conversion units and a pattern recognition unit. The red dates are recorded and weighed by a camera and a gravity sensor, The color signal of the red date is collected by the data acquisition unit and processed by the data processing unit, and then the collected electric signal is converted into a digital signal by the electric signal conversion unit to extract the color, longitudinal diameter, transverse diameter and shape of the object, Weight and other characteristics;

The input/output device comprises an input device, a display and a storage unit. The input/output device is used for inputting to the central processing unit. The storage unit is used for storing the digital signal outputted by the central processing unit. The display unit displays the internal detecting subsystem and the external detecting subsystem. The data; 
The wireless transmission subsystem comprises a wireless transceiver unit, a database, a data collation unit and a data collation unit. The data comparison unit is connected with a database, and the central processor and the database are wirelessly connected through a wireless transceiver unit. The central processor transmits the acquired digital signal Protocol data format through the wireless transceiver unit to the database, and through the database within the data comparison unit with the known date of internal quality information compared to the database and then send the results through the wireless transceiver unit to the central processor.

Compared with the prior art, the invention has the advantages that the red date quality detection system detects the internal and external quality of the red date through the internal detecting subsystem and the external detecting subsystem, and carries on the data comparison to the database through the wireless transmission subsystem, And finally through the display, can effectively filter the red date, simple structure, low production costs, which will promote the use of radiation through the magnetic field lines to the principles of red date to the internal rapid detection, and the detection of the signal output In order to facilitate the subsequent analysis.

\section{Extension experiment of non - destructive testing technology}

In the transformation of the results of the premise of the promotion, Ye Heyuan date industry companies to do a number of red date verification test, through the test found that the existence of differences in business and scientific research, and experience how we do research and practical experience. The experiment was conducted in seven groups, including the red date and gray date of the enterprise in 2014, the fresh red date and fresh red date which were picked at the end of September. The other three groups were 10 groups Jun date, Jun excellent 2, gray date. The sugar content comparison experiment was carried out for 8 groups, including the enterprises in 2014 mature period Jun red date, gray date, and the end of September picked fresh ash red date, fresh red date and 2013 processing of dry ash red date these five groups of samples; The remaining three groups were 10 groups of mature period Jun red date, Jun excellent 2, gray date. Among them, Junyou 2 and Jun 10 were selected for 14 samples because of sample reasons. The remaining 20 samples were selected for the experiment. Each group was numbered according to 1-14, 20-20, respectively. The first step, the first group of samples using near infrared spectroscopy for moisture and sugar content test, record each of the test value; the second step, each group is numbered odd number of samples, cut into thin strips using oven and the water content was determined by the method of drying weight loss. Then, from the remaining samples numbered even, each group used the GB method to measure the sugar separately and make 3 groups of parallel experiments to get the average value. In the third step, the average of the total sugar and water samples in each group were compared with the experimental data in the second step, and the results were obtained. The experimental data obtained by the two methods are available 
initially, and the moisture data measured by NIRS have high fitting with the data obtained by oven water measurement. From the data analysis of the instrument model, And the model of the situation when the sample is relatively high group, the two ways to test the data better fit, the error does not exceed 3 points; and the model does not contain samples of the two groups of data errors are in 5 Point, while the data from each group of samples of NIRS analyzer alone, apart from the individual differences between samples and samples, each group of data showed a corresponding stability. In the total sugar, the experimental data showed that the fit of the experimental data was very high, the error was less than 3 points, 10 of which were only 1 point error.

Near-infrared technology through the relevant demonstration, on-site exhibitors of red dates on the near-infrared technology has been further understanding and near-infrared technology had a keen interest. The prediction accuracy and stability of the sugar content and moisture correction model of red date in South Xinjiang were improved obviously by the related optimization and stability study of red date in south Xinjiang. The application and popularization of sugar content and water near infrared technology of red date in South Xinjiang Province played a positive role. But also for the late development of detection systems, improve the detection system provides a practical support to improve the competitiveness of red date in the market in southern Xinjiang will play a positive role in promoting, while other fruit quality detection technology in China has important practical significance. Temperature mixing correction model

In the process of promotion, red date users most value is the accuracy and stability of measurement, for which the process of promoting the adoption of the following technical means: a, single red dates A, B surface spectral scanning (simultaneous detection of two chemical values increase B) Temperature mixing model (for indoor and outdoor temperature measurement conditions) than the normal temperature model of the detection accuracy of 1 to 2 percentage points higher than the conventional one-point or batch scanning accuracy of 1 to 2 percentage points; In order to let the water evenly distributed, set up 4 different gradient test, 1 is 50 dates do not dry, 2 is 50 red date drying 2 hours, 3 is 50 red date drying 5 hours, 4 is 50 red date drying 10 hours, and the temperature was set at $80^{\circ}$ C. In order to obtain a stable value at different temperatures, the temperature factor is set. Near infrared spectra of red date were collected at $5{ }^{\circ} \mathrm{C}$, $15{ }^{\circ} \mathrm{C}$ (room temperature) and $35{ }^{\circ} \mathrm{C}$ for three different temperatures, and the moisture content of red date was measured by drying method.

After the model was established, the moisture and sugar content of the samples were measured. The average deviation between the moisture prediction value and the drying method was $0.75 \%$. The models of the model and the experimental prototype were tested by the factory manager and inspector. Very satisfied, the inspector Zhou Ping said that they can detect up to 300 samples a day, the water content of sugar, sugar can not detect the current workshop, and our experimental prototype in less than an hour to detect more than 100 samples of red date, not only can detect moisture But also can detect sugar content. In the workshop processing site inspectors deliberately in the pipeline to extract the 30 unacceptable to see the red dates, and 30 qualified red date with the experimental 
prototype for comparison. The results showed that the average water content of 30 unqualified red date fruits was $29.76 \%$ and the average sugar content was $66.54 \%$. The average water content of qualified red date was $22.28 \%$ and the average sugar content was $73.65 \%$. Ye Heyuan fruit industry standard is higher than $27 \%$ of the water content of red dates for the substandard goods, the test results and the actual quite consistent, the director and inspector of the model and experimental prototype of the test results are highly recognized.

\section{Conclusion}

In this paper, near infrared spectroscopy and chemometrics were used to analyze the characteristics of Red date in Nanjiang, the environmental factors of the laboratory and the performance of near-infrared spectroscopy. The near-infrared spectrometer was used to carry out the fine- Quality of water, total sugar and total acid and other characteristics of quality testing, compared with traditional methods to improve the detection accuracy. Near infrared diffuse reflectance spectra of red date were collected by using SupNIR-1520 (condenser technology, Hangzhou), and wavelength-dependent variables were selected by partial leastsquares method. Quantification of water, sugar content and total acid was established by various chemometrics methods Calibration model. The qualitative and quantitative parameters of red date in South Xinjiang were selected according to the method of chemometrics. On this basis, quantitative description of each index was established, including appearance quality factor and intrinsic quality characteristic factor. Quality factor, including the texture, fullness, size, aspect ratio, quality, color, gloss, etc; intrinsic quality characteristics of factors including sugar, acidity, water, vitamin $\mathrm{C}$ content. And then a single red dates of the image information, spectral information and composition of information packaged into a base element into an ACCESS database for easy retrieval and provide more quality dates information. By using the construction principle of the knowledge model, the near infrared spectroscopy analysis method and the visual programming technique were applied to the digital quality evaluation and design of the red date quality, and the difficult to express the product detection theory and technology through the relevant research and methods, Software, the preliminary completion of a comprehensive, intelligent features of the red date digital rapid detection and evaluation software for the quality of red date detection of digital and information technology has laid a foundation.

\section{Acknowledgement}

Project National Natural Science Foundation of China (NSFC): studies on the Dynamics Simulation on JUN Jujube Water and Nutrient Effects in Southern XinJiang.

Project number: 61462074 


\section{References}

[1] Liu Yande, Zhai Jianlong. Nearly infrared spectroscopy of soluble solids in navel orange. Journal of Northwest Sci - Tech University of Agriculture and Forestry, 7(1), pp. 18-23, 2013

[2] Zhang Bingfang, Yuan Libo, Zhang Bingxiu. Al. Studies on Total Acid and Soluble Sugar in Pulp of Tomato by Near Infrared Fiber Spectroscopy. Spectroscopy and Spectral Analysis, 3(2), pp. 32-35, 2014

[3] Li Guofang, He Jianbin.Design and implementation of "labor cost statistics system" based on C SHARP + ACCESS database. Information Security and Technology, 6(2), pp. 19-21, 2014

[4] Peng Yunfa, Peng Haigen. Nondestructive detection of water content in red date from south Xinjiang by near infrared spectroscopy.Food Science and Technology, 11(3), pp. 32-33, 2003.

[5] Wu Longguo, He Jianguo, He Xiaoguang. Progress of hyperspectral image technology in fruit nondestructive testing .Laser and Infrared, 9(4), pp.78-79, 2013 